\title{
7 Reference and Informativeness as cognitive processes in verbal communication
}

\begin{abstract}
Overspecification in reference is the provision of more information than is minimally required for a hearer to identify an intended referent, e.g., 'the stripy bowl' in the context of a single bowl. Since this kind of referring expression is not predicted by traditional accounts of reference, this chapter reviews research documenting the frequency of such expressions in various contexts. Drawing together recent empirical findings, it proposes reasons for overspecified reference from both the speaker's and the addressee's perspective. The pragmatic, cognitive and social significance of overspecification is discussed, and applications of research in this area are considered. We close by suggesting promising future directions for this strand of research.
\end{abstract}

Keywords: pragmatics, informativity, referring expressions, language production

\section{Introduction}

This chapter explores the relationship between human information processing and its realisation in language, specifically in referring expressions. It does so by investigating the processes at work when referring expressions contain more information than is strictly required (aka overspecification), and examines the interplay of this aspect of verbal behaviour with not fully manifest communication, i.e. meaning which is conveyed yet remains unsaid.

Reference relates to one of the most fundamental aspects of language; the fact that speakers use words to uniquely identify properties and entities in the world. For example, in a statement such as 'I like this', the pronoun 'I' is used to refer to the person who made this statement, and 'this' is used to refer to an entity or a property that the speaker has in mind. Even in this simple case though, it is clear that working out the referent of an expression is not a trivial matter. For example, uniquely identifying what is referred to by 'this' may involve a complex process taking into account the previous verbal context (what was the conversation about?), the physical context (is some particular entity visually or otherwise salient?), and the speaker's extra-linguistic behaviour (such as gestures and direction of eye-gaze) among other factors. Indeed, in many instances of communication there will be competing hypotheses about the referents of the words used. To make the case, knowing that the speaker is manifestly pointing at a ball a few centimetres away from her helps to narrow down the possible referents, but still, which aspect of the ball is she intending to identify? The colour? Texture? Size or shape? 
We may consider the fact that we do not routinely experience a breakdown of communication as a tribute to how good humans are in calibrating the use of referring expressions in such a way that their interlocutor can identify the intended referential target. As will become clear in the next section, there are rules that interlocutors tend to follow when producing and understanding referring expressions, and the study of these rules can reveal important aspects of the communicative system. As such, the study of reference is relevant to the study of the integration of information from multimodal sources in conversation (see this volume, chapter 17), the understanding of implicitly communicated meaning (see this volume, chapter 6), and the consideration of possible sources of misunderstanding in verbal communication (see this volume, chapter 24).

Our particular focus is on overspecification (also referred to as overinformativeness), an aspect of the referential use of language in which a speaker provides more information than is strictly necessary for a hearer to uniquely identify an intended target referent in a given situation. This aspect of communication is important because not only does overspecified reference affect comprehension processes, but it may also provide a window into some of the cognitive processes involved in speech production (e.g., the perception of certain features of the referent, comparison-making between referents and nonreferents in the linguistic and extralinguistic environment, and perspective-taking and the use of common ground). This chapter reviews some of the major findings from the adult processing literature with the aims of describing the incidence of overspecification and classifying some of its causal factors from both the speaker's and hearer's perspectives. It then discusses the pragmatic, cognitive and social implications of overspecification, applications for the research findings to date, and suggests future directions for this domain.

Within the language sciences, this topic spans subdisciplines of cognitive psychology, psycholinguistics, theoretical pragmatics, and the burgeoning field of experimental pragmatics (Noveck \& Sperber 2004; Noveck \& Reboul 2008; Katsos \& Cummins 2010; Katsos 2011, i.a.).

\section{Overspecification: Definitions and theoretical background}

When a speaker needs to point out a referent, she is faced with a range of constructions which could refer to the target entity. Broadly speaking, this referential choice may include an indefinite noun phrase, e.g., a ball, a demonstrative phrase, e.g., that ball, an anaphoric pronoun e.g., it, or a zero anaphor, e.g., there are two balls; a large $\emptyset$ and a small $\emptyset$. Standard explanations of how speakers select referential expressions have focused on cognitive status (e.g., Givón 1983; Ariel 1990; Gundel et al. 1993; Arnold 1998). When a referent is in focus or highly activated, a 'lighter' 
referential expression such as the pronoun it is licensed. Conversely, when the referent is less accessible, a more explicit form such as a definite description the ball is used. When a target referent is accompanied by another referent of the same type, a more explicit, modified noun phrase such as the large ball is required.

Restricting our review of the literature to studies investigating full noun phrases, experimental data challenge traditional accessibility accounts and support the notion that when speakers construct referring expressions, they frequently go beyond what is minimally required and refer to entities in more detail than is necessary for an addressee to uniquely identify them, e.g., referring to a target referent as the large ball in the presence of a single ball (Deutsch \& Pechmann 1982; Pechmann 1984, 1989; Mangold \& Pobel 1988; Maes, Arts and Noordman 2004; Ferreira, Slevc \& Rogers 2005; Engelhardt, Bailey \& Ferreira 2006; Barr 2007; Carbary \& Tanenhaus 2008). Frequently studied using the referential communication paradigm, speakers have been found to overinform in both laboratory-controlled and more naturalistic discourse. Referential communication tasks aim to elicit referring expressions (REs) which highlight one referent from an array of several others which usually share certain characteristics with the target. An overspecified RE is one which contains attributes over and above those minimally required for an addressee to identify the target referent uniquely. For example, a speaker referring to a large, red triangular target within an array also comprising a small blue triangle and a large red square, as the large red triangle is overinformative in the strict sense of including more information than is minimally required: either the large triangle or the red triangle would suffice in this case. Intuitively, speakers do not always produce minimally distinguishing REs, and it would seem computationally complex to do this as a complete online scan of the context and calculation of the distinguishing features would have to be undertaken upon producing any RE. As discussed below, overinforming may be causally linked to a variety of processes, including a lack of comparison activity between target and competitors.

Theoretically, overinformative referring expressions run contrary to Grice's second maxim of Quantity which states that speakers are expected to not give more information than is required (Grice 1975/1989), lest a pragmatic implicature be generated. This raises the question: what do hearers infer from these widely attested types of utterance? Answering this question requires an examination of the literature on contrastive inference (Sedivy et al. 1999; Grodner \& Sedivy 2011). However, there may be alternative consequences of overinforming than pragmatic implicature, e.g., speeding up a hearer's search for the referent (Mangold \& Pobel 1988; Arts 2004, 2011), or reducing the risk of the signal being lost (Shannon \& Weaver 1949).

Within the theory of Conversational Implicature (1975/1989), Grice conceded that a violation of Quantity-2 or in plain terms, saying too much, was not as serious a cause for concern (i.e. would not as readily produce implicatures) as violating other maxims and at the very least, might be 'merely a waste of time' (1989: 26). 
However, we argue that overinformative utterances do impact on online interpretation. The psychological investigation of referential overspecification is becoming a major research thread within the linguistic and cognitive sciences, furthering the original philosophical contemplation of reference in human communication.

\section{The incidence of overspecification in production}

Just as overinforming is commonplace in the wild (consider the rich detail given in narrative fiction or in precise instruction), studies from experimental linguistics have revealed high rates of overinformativeness. Early research revealed that in referential communication tasks, $28 \%$ of adults' expressions were overinformative (Deutsch \& Pechmann 1982), and in a similar experiment $60 \%$ were found to be so (Pechmann 1984). In a recent study, Koolen et al. (2011) found that around $50 \%$ of speakers' referring expressions in their study contained more information than needed for unique identification of the target. Engelhardt, Bailey \& Ferreira (2006) found that speakers not only supplied modifiers in descriptions when they were necessary for disambiguation between multiple referents, but they also supplied them in single-referent conditions in around a third of referring expressions (though see Davies \& Katsos 2013). Cross-linguistically, speakers of Yucatec Maya have been found to overspecify at very similar levels (33\%: Butler et al. 2011).

One observation from this small sample of research, as well as from the broader survey below is the variable rates of overspecification found in the data. In the following analysis of representative studies, we find rates of overspecification ranging from $0 \%$ to around $60 \%$. This can be tentatively explained by the fact that there are various and interacting contextual factors which impact on the choice of $\mathrm{RE}$ (see recommendations for further research below). It has been argued that speakers design their REs for efficient identification on the hearer's part (Arnold 2008), but lower-level speaker-oriented processes may also be at work. The following discussion is divided by proposed speaker-oriented and hearer-oriented motivations for overinforming.

\section{Speaker-oriented reasons for overinforming}

\subsection{Lack of comparison activity}

Felicitous referring expressions must not merely describe indiscriminate features of a target referent, but crucially, the ways in which the target differs from nonreferents. The act of comparing referents to nonreferents is critical in formulating REs, and affects where they fall on the cline between underinformativeness/ambiguity, 
through minimal contrastiveness, to overinformativeness. Based on the assumption that speech becomes more efficient developmentally, Freedle (1972) proposed the Minimal Redundancy Hypothesis, which held that adults' REs would contain the minimum amount of features required to distinguish the referent from the nonreferent. However, Freedle's data did not support the hypothesis, as adult participants included more features than were minimally required for unique identification, especially where there were higher numbers of nonreferents and feature-types in an array. It seems that although adult speakers engage in comparison activity, their descriptions do not follow in the most efficient way. As overinformativeness is more common when dealing with complex arrays, a resource-sharing account would suggest that overinforming requires fewer processing resources. Longer, allinclusive descriptions are easier to produce due to a lack of comparison activity, especially when cognitive resources are being spent elsewhere (i.e. in linguistic production processes). Relatedly, as referring and resolution is a collaborative process, speakers can produce their descriptions in the knowledge that their hearer can indicate if the expression is inadequate, reinforcing the knowledge that a full scan is not compulsory, particularly in highly interactive discourse contexts (cf. Clark \& Wilkes-Gibbs 1986).

Pechmann (1989) found that amongst his adult sample, $21 \%$ of REs were overspecified, and suggested that it is the incremental nature of speech processing which drives overspecification: adults start speaking before they have scanned the whole array and deduced the distinguishing features of the target referent (rendering the early part of the RE noncontrastive). In this study, the overspecified part of the description was almost always colour. Since colour is an absolute rather than a scalar attribute, it can be articulated without looking to nonreferents. Under this account, the process of generating referring expressions would first involve object location, then a simultaneous calculation and articulation of distinctive features. Thus, one potential cause of overspecification is incremental/incomplete scanning and comparison activity before articulation begins. Because it bypasses the need to undertake detailed and precise comparison activity, overspecification requires fewer cognitive resources. It also appears that lack of scan may be more likely in complex or highly interactive discourse situations.

With the availability of eye tracking techniques, it is now possible to examine the relationship between visual scene interrogation and articulation of perceived attributes (and in turn, how the conventional ordering of attributive adjectives in a particular language may constrain both the searching and articulatory processes). The literature to date indicates that lack of complete scan may result in overinformative REs, but more work is needed in this domain to draw firmer conclusions.

\subsection{Perceptual salience}

Certain attributes appear more frequently in overspecification. Notably, it was colour which was the overspecified attribute in $98 \%$ of cases of overspecification in 
Pechmann's 1989 work. In addition to being central to the creation of a gestalt, colour is high on the perceptibility hierarchy by virtue of quick processing by the visual system (Arts 2004, 2011), which may lead to quick encoding or lack of attribute suppression in REs. Mangold \& Pobel (1988) ran a referential communication task, wherein a target from an array of 24 shapes differing along colour, size and shape dimensions was referred to by speakers. The marked target could be described by mentioning a totally discriminating feature (an attribute shown by the target only), a partially discriminating feature (shared by some of the competitors), or a totally nondiscriminating features (shared by all of the competitors). Two major referential patterns emerged: partially discriminative features were more common in overspecifications than nondiscriminating features, and the chances of overspecification were increased when the totally discriminating feature was relatively low in perceptual salience (i.e. size or shape). Thus, colour attributes are commonly provided even when they do not function as a distinguishing feature. Colour terms may be so commonly overspecified that they function in a fundamentally different way to other adjectives. For example, they are not interpreted contrastively, as shown by the lack of an early bias in referent identification, cf. the strong early effects found on processing scalar terms (Sedivy et al. 1999).

Carbary \& Tanenhaus (2008) tested the hypothesis that the presence of an attribute in an array-competitor would increase the incidence of overspecified REs. Their arrays consisted of one target and three unrelated items (no shared attributes) in one condition, and another condition in which one of the competitors shared an attribute of the target (e.g., striped cat and a striped shirt). In the first, they found $11 \%$ overspecification, rising to $25 \%$ in the second, despite the modifier not helping identification any faster than a bare noun would (the control condition containing a contrast set yielded $90 \%$ modification). These results suggest that the salience of a particular attribute is increased by the presence of that attribute in a competitor. Thus, that attribute is more accessible, increasing the likelihood of that adjective surfacing in the referring expression.

\section{Hearer-oriented reasons for overinforming}

\subsection{Facilitation of the hearer's reference resolution}

In encountering a salient yet noncontrastive attribute such as colour, a hearer is able to zone in on a subset of a larger array. This is supported by evidence from Mangold and Pobel (1988), who found that overspecification shortened identification time if the redundant feature was better perceptible than the discriminating feature. The utility (and thus incidence) of this type of overinformativeness may be increased when contexts are complex or otherwise demanding (Paraboni et al. 2007), and the cooperative speaker may be motivated to reduce the search space 
for the hearer. Arts $(2004,2011)$ found that certain types of overspecification almost consistently led to accelerated resolution, especially attributes concerning the location of an object. Hypothetically, since speakers do not always know which features the listener might notice, an overinformative 'scattergun' approach may increase the likelihood of the target being hit, i.e. the features mentioned match the features noticed. Consistent with this view is the finding that the number of choices available to a speaker when referring affects specification levels, e.g., references to people (which vary more in terms of the potential attributes which could be used in a $\mathrm{RE}$ ) are more likely to be overspecified than references to furniture (Koolen et al. 2011). Conversely, when arrays are highly simplified, rates of overspecification fall (Davies \& Katsos 2009, 2013).

This type of cooperative behaviour is one of the hallmarks of pragmatic communication. Following Grice (1989), REs should be adequate, efficient and sensitive to hearer needs (Dale 1992). Speakers assess the common ground and judge what their hearer needs to know. In discourse contexts with minimal common ground, speakers may include redundant information to convey salience or relevance, e.g., This is Jacqui, my boss and to reduce the cognitive effort in resolving reference (Sperber \& Wilson 1986/1995).

Thus, speakers help out their interlocutors by providing modifiers which apply to more than one referent, but crucially rule out some of the items in complex arrays. Speakers adhere to listener expectation or discourse convention, and may provide information which is not referential in function, but in some other way communicative or pragmatically felicitous. Supporting the notion that overspecification is a truly hearer-oriented mechanism, Ferreira, Slevc \& Rogers (2005) found their speaker-participants to overspecify more when speaking to an actual listener than a hypothetical one.

\subsection{Overspecification as a function of discourse goals}

Certain types of discourse with their corresponding goals correlate with characteristic levels of overspecification. It is not entirely clear whether this factor is a speaker- or hearer-oriented process, but in line with our general emphasis on cooperation in referential communication, we frame this factor as a means of lightening the processing load for the hearer, or at least increasing the chances of the hearer interpreting the speaker's meaning as intended.

Maes, Arts \& Noordman (2004) report an elegant study which chimes with accounts of referential choice based on the accessibility of the referent (Ariel 1990, i.a.). The framework is expanded by asserting that levels of informativeness interact with discourse goals, and a fine-grained analysis of perceptual, propositional and anaphoric overspecification reveal a correlation between overspecification and discourse function. When speakers used information to teach a hearer a long-term skill, there was more overspecification than when a hearer only had to execute 
the action once. Further, when the speaker and hearer could see the same array (remotely), there was more perceptual overspecification as presumably the speaker was concerned with ensuring that both parties had the same referent in mind before going on to issue more complex commands (strengthened by Clark \& WilkesGibbs' Principle of Distant Responsibility 1986). Thus, in addition to the premise that informativeness and activation are inversely correlated, it seems that task demands strongly affect the choice of RE. Maes et al.'s work also highlights the role of fault-criticality: overspecification is indeed expected in technical contexts where precision is prioritised and the risk of misunderstanding is high. In line with Information Theory, redundancy safeguards loss of information (Shannon \& Weaver 1949).

\subsection{Apparent overspecification: Speakers contrast between current and previous contexts as well as within a given array}

After reviewing the literature on speaker- and hearer-oriented mechanisms for overinforming, an important methodological consideration should be considered in order to provide an accurate picture of the nature of referential overspecification. Much of the research on referential behaviour takes an ahistorical view of contrastiveness, i.e. comparing nonreferents to target referents within the concurrent array only. Pechmann (1984) observed that REs do not only relate to the concurrent visual array, but also to how the referent is distinct from entities of the same overall type in previously encountered arrays, which leave a trace in subsequent REs. Speakers were found to stress the modifier which contrasted with the directly preceding target, e.g., a yellow flower, then a green flower, even in arrays where there were no other flowers concurrently present (thus the modifier would not be strictly necessary from an ahistorical point of view). This data suggests that the prior linguistic contrast rather than concurrent visual contrast takes prosodic priority.

Although historical contrast has been classified as a speaker-oriented process since the inclusion of the previous contrast is not useful as a contrast for the hearer (Koolen et al. 2011), this referential strategy may in fact be designed to help the listener. Pechmann (1984: 428) discusses holistic representations of objects in the mind of the hearer, after Deutsch (1976) reported faster locating of objects when hearers received redundant object descriptions than minimally contrastive ones. Theoretically, the hearer is thought to construct a complete representation of the object upon hearing its description, and the more precise the description (and thus the representation), the faster the identification process. Further, such representations persevere across subsequent encounters with different objects, so by mentioning (or stressing) the feature which contrasts with objects encountered directly beforehand, the hearer is implicitly told to adjust only that one feature, and to use 
the existing representation for all other identificational details. Clearly an economical means of processing, the importance of this endophoric (i.e. discourse-internal) contrast was later endorsed by Levelt (1989) as gestalt creation. There may be additional reasons as to why cross-trial relationships should take priority in features mentioned: endophoric information is more strongly established in the interlocutors' common ground whilst exophoric (i.e. nonlinguistic/visual) is less so. Further, the endophoric relationship means that there are fewer competitors to be processed (by definition, there is only one preceding target object). Finally, accessing shortterm memory may be quicker than processing the visual array (Pechmann 1989).

More recent work has furthered the idea of overspecification as a result of cross-trial comparison-making. Labelled by Dale \& Reiter (1995) as reference scripts, previously used sets of features and values may be used by speakers in subsequent REs, even if they are not contrastive in a given array. Whilst in line with work on conceptual pacts (Clark \& Wilkes-Gibbs 1986; Metzing \& Brennan 2003), predictions from a reference script approach were not realised by some of the older literature, e.g., Pechmann (1989: 106) wherein speakers did not produce lists of feature-value clusters for consecutive descriptions. However, Brennan and Clark's (1996) work on lexical entrainment shows that increasing levels of specification persevere over time, even when concurrent arrays do not demand a higherlevel term than the basic-level RE. For example, when a loafer was shown in an array without any other shoes, it is labelled a shoe, then when it appears alongside other types of shoes, it is called a loafer. Crucially, when it then appears within its original array, speakers retain the higher-level term loafer. Barr (2007) also found strong automatic memory effects on REs. In conditions where speakers had previously referred to shapes using modification (optimally informative in the initial contexts), they maintained the referring strategy on later trials with new addressees and in contexts where modification was not required. It seems speakers find it hard to revert to basic level terms once they have elevated the level of description to a more specific taxonomy. This may be due to recent memory traces overriding the more effortful strategy of audience design.

This wider view of discourse context reveals that overspecification may only be apparent rather than truly overinformative. To recap, if an analysis of referential context is restricted to judging minimal/redundant contrastiveness in relation to other items in the concurrent context, speakers might appear to be overinformative. However, if the field of view is broadened to include the preceding temporal context which is inarguably part of the discourse model, modifiers may be functioning as endophoric contrasts across time. Previously used features persevere over time to leave a trace both in the features used (i.e. modifier types such as height or size) and the level of specification required. There are long-term effects of overspecification which bias speakers to retain the level of previously-required specificity even when the subsequent arrays do not demand such levels of modification. 


\section{Interim summary}

The review and interpretation of the literature above suggests that a) interlocutors do not adhere to expectations to be optimally informative and instead frequently provide modification beyond the minimal amounts required to resolve reference, and $b$ ) there are various factors which may lead to overspecification, both speakeroriented and hearer-oriented. For the former set of processes, we propose that lack of comparison activity during the speaker's message formulation, and the perceptual salience of overspecified attributes may lead to the articulation of information beyond minimal contrastiveness. Hearer-oriented factors may also play a role in the inclusion of overinformative referential material, e.g., the desire to facilitate the hearer's reference resolution. Communicative goals of the discourse situation may also influence the speaker's inclusion of 'extra' material since redundancy increases the chances of complex information being interpreted as intended. Finally, adherence to previously established levels of informativeness or to specific referential pacts may also be realised as overspecified reference, and speakers may subtly acknowledge contrasting referents from previously encountered aspects of the discourse context, leading to apparent overinformativeness with reference to the concurrent array.

\section{The pragmatic significance of overspecification}

From the disparate factors which underlie overspecification, general pragmatic mechanisms emerge. Pragmatic cooperation is a driving factor in referential pacts, facilitation of reference resolution, and adjustments according to discourse goals. Indeed, since referring is by definition a collaborative process, it would be difficult to conceive of pragmatic considerations being divorced from the underlying mechanisms.

The central pragmatic inference generated by modified definite descriptions is contrastive inference (CI), by which hearers infer meaning about the referential context due to modification of the target referent. Referential overspecification enriches the discourse context, but rather than adding to the representation of the referent in focus, it enriches the backdrop in which the referent is placed. For example, on hearing Pass me the big book, a hearer generates a CI that there must be a contrasting (i.e. smaller) book elsewhere in the context, as he infers a rational motivation for the speaker's inclusion of the modification within a defined referential context. Gricean accounts of CI state that such inferences are drawn due to REs deviating from a default form which would conventionally be used (and expected to be used) to refer to a sole entity of a given type. Sedivy and colleagues (Sedivy et al. 1999; Sedivy 2003) show that in the presence of a contrast set (e.g., a tall and 
a short glass) and a competitor to the tall glass (e.g., a tall jug), hearers fixate on the tall glass on hearing 'tall' and before they hear the disambiguating noun ('glass' or 'jug'). Hearers thus engage in Gricean inferencing; they reason that if the speaker meant to refer to the tall jug, they should have referred to it without a modifying adjective in order to avoid overinformativeness. Instead, the only object they should refer to with a modifying adjective is the one for which there is a contrast-mate, i.e. the tall glass, where omission of the modification would lead to under-informativeness. In this way, Gricean reasoning facilitates and speeds referent resolution thanks to modifiers providing early cues as to the identity of the referent.

On hearing an utterance which appears to deviate from the expected amount of information, a hearer works to enrich the context which will in turn reinstate the assumption of the speaker's adherence to the second maxim of Quantity. The context is expanded to include the presence of a contrast-mate to accompany the referent of the modified utterance. Furthermore, by uttering the scalar adjective, a speaker assumes that the hearer has noticed the presence of multiple glasses, and noticed that they differ along a scale of tallness. In the process of formulating a modified utterance, the speaker also realises that in order for the hearer to identify the intended target, they as speakers need to provide enough information for the hearer to identify the one which best fits the expression provided. This assumption is in the spirit of Relevance Theory (Sperber \& Wilson 1986/1995), in that it acknowledges that a speaker will take account of the hearer's current cognitive state. In the referential world, a hearer's cognitive state can be partially inferred from common ground, often from a shared visual field. Thus, the overspecification of modified REs in the context of a single item of a specific type should involve greater processing effort as the hearer looks for some relevance of the modifier once they have appropriately discarded a contrastive function.

\section{The cognitive significance of overinformativeness}

Referential overspecification may result from incomplete consideration of the common ground which exists between interlocutors. For example, if a speaker can see a small and a large glass, but the hearer can only see the large glass, then the modified RE the large glass would be overspecified for the hearer. In formulating a message, cooperative speakers should acknowledge what is shared by their addressee and construct their utterance accordingly. In doing so, the speaker engages in mentalising activity. Empirical research investigating this phenomenon has typically used referential communication tasks in which the speaker's and the hearer's views are different, and thus the speaker has to integrate the common ground (which contrasts with their own privileged ground) before articulating a felicitous referring expression (Keysar, Barr \& Horton 1998; Keysar et al. 2000; Nadig \& Sedi- 
vy 2002; Hanna, Tanenhaus \& Trueswell 2003; Hanna \& Tanenhaus 2004; Tanenhaus \& Brown-Schmidt 2008, i.a.).

Keysar, Lin and Barr (2003) claimed that although adults have a fully-fledged Theory of Mind (ToM), they may not reliably and automatically apply it. They argue that classic ToM tests probe metacognitive, reflective ToM (e.g., 'where does she think the chocolate is?' in the prototypical Sally-Anne task) rather than its spontaneous usage. In a referential communication task, hearer-participants were directed to move objects around a frame. Before beginning the game, hearers hid an object inside a bag and placed it in the frame. Occasionally, the item in the bag more closely fitted the description which a speaker gave than a mutually visible object, and although hearer-participants knew that the speaker did not know what was in the bag, they often took it to be the intended referent. These results show a strong dissociation between the ability to reflectively distinguish one's own beliefs from others', and the routine use of this ability in interaction. It is possible therefore that overinformative speakers are behaving egocentrically because they are not deploying their ToM abilities online. Nadig and Sedivy's (2002) results are compatible with this view, as the children in their study showed the ability to use ToM to perspective-take when they must (i.e. when faced with ambiguity, and consciously forming a strategy). Crucially, the overinformative children may be acting spontaneously, i.e. without recourse to ToM, in line with Keysar et al.'s (1998) Monitoring and Adjustment model (though see Rubio-Fernandez 2008 for a critique of these studies).

As mentioned in our discussion of perceptual salience and overspecification, visual processing and optimal linguistic encoding may compromise each other through a sharing of cognitive resources. When arrays are more complex and the number of targets increases, activation may be lower to the target(s) in question. As a result of this, full comparison activity between the target and the nonreferents may be sacrificed, leading to greater overspecification. Such an account is put forward by Koolen et al. (2011) who found plural targets REs to be more frequently overspecified than single target REs, and similarly by Arnold and Griffin (2007) who found more full NP expressions than appropriate pronominal expressions to plural targets.

In the domain of general cognitive mechanisms, recommended areas for future investigation would be the suppression of speaker-salient features of the referent, and the nature of comparison-making by speakers between referents and nonreferents.

\section{The social significance of overinformativeness}

Inherent in the observation that referring is a fundamentally pragmatic process is the fact that it is also a social phenomenon. Since expressions themselves do not 
refer, it is the user of those words (i.e. the speaker) who refers (Strawson 1950). As a corollary the reference does not go through without an addressee. Thus by nature, reference and its implicit informativeness is a social activity. From very early in development (around 9 to 12 months of age), children are motivated to share entities and activities with others in so-called triadic interactions (Carpenter, Nagell \& Tomasello 1998). Even infants' prelinguistic referential communication (e.g., pointing) is clearly a social act (Bates, Camaioni \& Volterra 1975; Franco \& Butterworth 1996; Liszkowski et al. 2004; Liszkowski, Carpenter \& Tomasello 2007a; 2007b), a communicative intention which continues into adulthood.

\section{Applications of research into overspecification}

Fields outside of pragmatics have made helpful contributions to investigations of overspecification. Computational linguistic research has documented high rates of overinforming in human speech (Dale \& Reiter 1995; Viethen \& Dale 2006; Paraboni et al. 2007; van der Sluis \& Krahmer 2007; Viethen et al. 2008). This dialogue between psycholinguistics and computational linguists has been mutually beneficial: ${ }^{1}$ how speakers choose the content and form of their REs is not only of interest to psycholinguists and philosophers of language, it is a pervasive challenge for natural language generation systems. Such systems aim to approximate the types of utterances that human speakers produce in dialogue to single out a target object from other objects in an array. The descriptions produced by computational algorithms have made substantial progress in achieving minimal contrastiveness in machine-generated speech, e.g., the full brevity algorithm (Dale 1989; 1992) and the incremental algorithm (Dale \& Reiter 1995; van Deemter 2002) but in light of the frequent overinformativeness found in naturalistic human speech in authentically complex contexts, there remains a quantitative and qualitative gap between human and computer-generated speech. Corpus-based and experimental methods have recently been used to evaluate computational models against human-produced data (Gatt et al. 2007; Gatt \& Belz 2008; Viethen \& Dale 2008), and further collaboration between psycholinguists and computational linguists is keenly encouraged (van Deemter et al. 2009), particularly to inform questions around audience design, interactivity, multimodality, common ground, and under- and overinformativeness.

1 See the many publications emerging from the NWO Vici project, 2008-2013: Bridging the gap between psycholinguistics and computational linguistics: The case of referring expressions: http:// bridging.uvt.nl/ 


\section{Future directions}

Research on overspecification is in robust health across linguistics, psychology and computer science, benefiting from its interdisciplinarity. Whilst production of overinformativeness has been measured and analysed across many studies dating from the early 1980s, the impact of this pragmatic phenomenon is yet to be fully explored from the comprehender's perspective. Future research should use experimental methods which measure not only the behavioural but also the neuropsychological effects of processing these types of utterances (e.g., Engelhardt, Demiral \& Ferreira, 2011).

At the beginning of the chapter, we proposed that there may be multiple interacting contextual factors which impact on the choice of RE. One key recommendation for future research is to examine which factors inherent in discourse/experimental tasks invoke higher and lower rates of overspecification, as a means of tracing causal patterns for the attested rates of overspecification. One could start by examining the interaction of visual and linguistic processing. The emerging finding that overinformativeness increases when aspects of the visual display are made more salient addresses the need to delineate the conditions under which human speakers overspecify.

Beyond the scope of this chapter is the large literature on the development of reference which lends useful insights from children's use of overspecified reference to the current debate (e.g., Flavell et al. 1981; Dickson 1982; Sonnenschein \& Whitehurst 1984; Revelle et al. 1985; Lloyd, Mann \& Peers 1998; Matthews et al. 2006, 2007; Huang \& Snedeker 2008; Davies \& Katsos 2010; Nilsen \& Graham 2012; Bannard, Klinger \& Tomasello 2013; Morisseau, Davies \& Matthews 2013). We expect that future endeavours will continue in tandem with these areas.

\section{References}

Ariel, Mira. 1990. Accessing noun-phrase antecedents. London: Routledge.

Arnold, Jennifer E. 1998. Reference form and discourse patterns. Stanford: Stanford University dissertation.

Arnold, Jennifer E. \& Zenzi Griffin. 2007. The effect of additional characters on choice of referring expression: Everyone counts. Journal of Memory and Language 56. 521-536.

Arnold, Jennifer E. 2008. Reference production: production internal and addressee-oriented processes. Language and Cognitive Processes 23(4). 495-527.

Arts, Anja. 2004. Overspecification in Instructive Texts. Tilburg University dissertation.

Arts, Anja, Alfons Maes, Leo Noordman \& Carel Jansen. 2004. Reference management in instructive discourse. Discourse Processes 37(2). 117-144.

Arts, Anja, Alfons Maes, Leo Noordman \& Carel Jansen. 2011. Overspecification facilitates object identification. Journal of Pragmatics 43(1). 361-374. 
Bannard, Colin, Jörn Klinger \& Michael Tomasello. 2013. How Selective are 3-year-olds in Imitating Novel Linguistic Material? Developmental Psychology, 49(12) 2344-64.

Barr, Dale J. 2007. Audience design and automatic processing in reference generation. Poster presented at Architectures and Mechanisms of Language Processing (AMLaP), Turku, August, 2007.

Bates, Elizabeth, Luigia Camaioni \& Virginia Volterra. 1975. The acquisition of performatives prior to speech. Merrill-Palmer Quarterly 21. 205-226.

Butler, Lindsay K., T. Florian Jaeger, Juergen Bohnemeyer \& Katrina Furth. 2011. Learning to Express Visual Contrasts in the Production of Referring Expressions in Yucatec Maya. Proceedings of the PRE-CogSci 2011 workshop: Bridging the gap between computational, empirical and theoretical approaches to reference, Boston, July $20^{\text {th }}$.

Carbary, Kathleen M. \& Michael Tanenhaus. 2008. Syntactic priming and message encoding in unscripted dialogue. Paper presented at Architectures and Mechanisms of Language Processing (AMLaP), Cambridge, September 2008.

Carpenter, M., Nagell, K. \& Tomasello, M. 1998b. Social cognition, joint attention, and communicative competence from 9 to 15 months of age. Monographs of the Society for Research in Child Development 63. 176.

Clark, Herbert H. \& Deanna Wilkes-Gibbs. 1986. Referring as a collaborative process. Cognition 22. 1-39.

Dale, Robert. 1992. Generating Referring Expressions: Constructing Descriptions in a Domain of Objects and Processes. Cambridge, MA: MIT Press.

Dale, Robert \& Ehud Reiter. 1995. Computational interpretations of the Gricean maxims in the generation of referring expressions. Cognitive Science 19. 233-263.

Davies, Catherine \& Napoleon Katsos. 2009. Are Interlocutors as Sensitive to Overinformativeness as they are to Under-informativeness? In Kees Van Deemter, Albert Gatt, Roger PG van Gompel \& Emiel Krahme (eds.), Proceedings of the Workshop on the Production of Referring Expressions: Bridging the gap between computational and empirical approaches to reference (PRE-CogSci 2009), Amsterdam, 29 July 2009.

Davies, Catherine \& Napoleon Katsos. 2010. Over-informative children: Production/ comprehension asymmetry or tolerance to pragmatic violations? Lingua 120(8). 1956-1972.

Davies, Catherine \& Napoleon Katsos. 2013. Are speakers and listeners 'only moderately Gricean'? An empirical response to Engelhardt, Bailey and Ferreira (2006). Journal of Pragmatics 49. 78-106.

Deutsch, Werner. 1976. Sprachliche Redundanz und Obektidentifikation. Marburg dissertation.

Deutsch, Werner \& Thomas Pechmann. 1982. Social-interaction and the development of definite descriptions. Cognition 11(2). 159-184.

Dickson, W. Patrick. 1982. Two decades of referential communication research: A review and meta-analysis. In Charles J. Brainerd \& Michael Presley (eds.), Verbal Processes in Children, 1-33. New York: Springer Verlag.

Engelhardt, Paul E., Karl GD Bailey \& Fernanda Ferreira. 2006. Do speakers and listeners observe the Gricean Maxim of Quantity? Journal of Memory and Language 54(4). 554-573.

Engelhardt, Paul E., S. Demiral \& Fernanda Ferreira. 2011. Over-specified referring expressions impair comprehension: An ERP study. Brain and cognition 77(2). 304-314.

Ferreira, Victor S., L. Robert Slevc \& Erin S. Rogers. 2005. How do speakers avoid ambiguous linguistic expressions?. Cognition 96(3). 263-284.

Flavell, John H., James Ramsey Speer, Frances L. Green, Diane L. August \& Grover J. Whitehurs. 1981. The development of comprehension monitoring and knowledge about communication. Monographs of the Society for Research in Child Development. 1-65.

Franco, Fabia \& George Butterworth. 1996. Pointing and social awareness: Declaring and requesting in the second year. Journal of Child Language 23. 307-336. 
Freedle, Roy. 1972. Language users as fallible information-processors: Implications for measuring and modelling comprehension. In Roy Freedle \& Joy Carroll (eds.), Language comprehension and the acquisition of knowledge 169-209. Washington DC: Winston.

Gatt, Albert \& Anja Belz. 2008. Attribute selection for referring expression generation: New algorithms and evaluation methods. Proceedings of the $5^{\text {th }}$ International Conference on Natural Language Generation, INLG-08.

Gatt, Albert, lelka Van Der Sluis \& Kees Van Deemter. 2007. Evaluating algorithms for the generation of referring expressions using a balanced corpus. Proceedings of the $11^{\text {th }}$ European Workshop on Natural Language Generation, ENLG-07.

Givón, Talmy. 1983. Topic Continuity in Discourse: An Introduction. In Talmy Givón (ed.), Topic Continuity in Discourse: A Quantitative Cross-language Study, 1-42. Amsterdam: John Benjamins Publishing.

Grice, H. Paul. 1975. Logic and conversation. In Peter Cole \& Jerry Morgan (eds.), Syntax and semantics, Vol. 3: Speech Acts, 41-58. New York: Academic Press.

Grodner, Daniel \& Julie Sedivy. 2011. The effects of speaker-specific information on pragmatic inferences. In Neal Pearlmutter \& Edward Gibson (eds.), The Processing and Acquisition of Reference. Cambridge, MA: MIT Press.

Gundel, Jeanette K., Nancy Hedberg \& Ron Zacharski. 1993. Cognitive status and the form of referring expressions in discourse. Language 69(2). 274-307.

Hanna, Joy E., Michael K. Tanenhaus \& John C. Trueswell. 2003. The effects of common ground and perspective on domains of referential interpretation. Journal of Memory and Language 49(1). 43-61.

Huang, Yi Ting \& Jesse Snedeke. 2008. Use of referential context in children's language processing. Proceedings of the $30^{\text {th }}$ annual meeting of the Cognitive Science Society, Washington, DC. 1381-1385.

Katsos, Napoleon. 2011. Experimental Investigations and Pragmatic Theorising. In Keith Allan \& Kasia Jaszczolt (eds.), The Cambridge Handbook of Pragmatics, 275-290. Cambridge University Press.

Katsos, Napoleon \& Chris Cummins. 2010. Pragmatics: From Theory to Experiment and Back Again. Language and Linguistics Compass 4(5). 282-295.

Keysar, Boaz, Dale J. Barr \& William S. Horton. 1998. The egocentric basis of language use: Insights from a processing approach. Current Directions in Psychological Science 7(2). 4650.

Keysar, Boaz, Dale J. Barr, Jennifer A. Balin \& Jason S. Brauner. 2000. Taking perspective in conversation: The role of mutual knowledge in comprehension. Psychological Science 11(1). 32-38.

Keysar, Boaz, Shuhong Lin \& Dale J. Barr. 2003. Limits on theory of mind use in adults. Cognition 89(1). 25-41.

Koolen, Ruud, Albert Gatt, Martijn Goudbeek \& Emiel Krahmer. 2011. Factors causing overspecification in definite descriptions. Journal of Pragmatics 43(13), 3231-3250.

Levelt, Willem. 1989. Speaking. London: The MIT Press.

Liszkowski, Ulf, Malinda Carpenter, Anne Henning, Tricia Striano \& Michael Tomasello. 2004. Twelve-month-olds point to share attention and interest. Developmental Science 7. 297-307.

Liszkowski, Ulf, Malinda Carpenter \& Michael Tomasello. 2007a. Pointing out new news, old news, and absent referents at 12 months. Developmental Science 10(2). F1-F7.

Liszkowski, Ulf, Malinda Carpenter \& Michael Tomasello. 2007b. Reference and attitude in infant pointing. Journal of Child Language 34(1). 1-20.

Lloyd, Peter, Sandi Mann \& Ian Peers. 1998. The growth of speaker and listener skills from five to eleven years: a normative study. First Language 18(1). 81-103.

Mangold, Ronald \& Rupert Pobel. 1988. Informativeness and instrumentality in referential communication. Journal of Language and Social Psychology 7. 181-191. 
Matthews, Danielle, Elena Lieven, \& Michael Tomasello. 2007. How toddlers and preschoolers learn to uniquely identify referents for others: A training study. Child Development 78. 17441759.

Matthews, Danielle, Elena Lieven, Anna Theakston \& Michael Tomasello. 2006. The effect of perceptual availability and prior discourse on young children's use of referring expressions. Applied Psycholinguistics 27. 403-422.

Metzing, Charles \& Susan E. Brennan. 2003. When conceptual pacts are broken: Partner-specific effects on the comprehension of referring expressions. Journal of Memory and Language 49. 201-213.

Morisseau, Tiffany, Catherine Davies \& Danielle Matthews. In press. How do 3- and 5-year-olds respond to over- and under-informative utterances?. Journal of Pragmatics 59. 26-39.

Nadig, Aparna S. \& Julie C. Sedivy. 2002. Evidence of perspective-taking constraints in children's on-line reference resolution. Psychological Science 13(4). 329-336.

Nilsen, Elizabeth S. \& Susan A. Graham. 2012. The development of preschoolers' appreciation of communicative ambiguity. Child Development 83(4). 1400-1415.

Noveck, Ira A. \& Anne Rebou. 2008. Experimental Pragmatics: A Gricean turn in the study of language. Trends in Cognitive Sciences 12(11). 425-431.

Noveck, Ira A. \& Dan Sperber. 2004. Experimental Pragmatics. Basingstoke: Palgrave Macmillan.

Paraboni, Ivandré, Kees Van Deemter \& Judith Masthoff. 2007. Generating referring expressions: Making referents easy to identify. Computational Linguistic 33(2). 229-254.

Pechmann, Thomas. 1984. Accentuation and Redundancy in Children and Adult's Referential Communication'. In Herman Bouma, \& Don G. Bouwhuis (eds.), Attention and Performance X: Control of Language Processes. NJ: Erlbaum.

Pechmann, Thomas. 1989. Incremental Speech Production and Referential Overspecification. Linguistics 27(1). 89-110.

Revelle, Glenda L., Henry M. Wellman \& Julie D. Karabenick. 1985. Comprehension monitoring in preschool children. Child Development 56. 654-663.

Rubio-Fernandez, Paula. 2008. On the automaticity of egocentricity: A review of the Egocentric Anchoring and Adjustment model of perspective taking. UCL Working Papers in Linguistics 20. 247-274

Sedivy, Julie. 2003. Pragmatic versus form-based accounts of referential contrast: Evidence for effects of informativity expectations. Journal of Psycholinguistic Research 32(1). 3-23.

Sedivy, Julie C., Michael K. Tanenhaus, Craig G. Chambers \& Gregory N. Carlson. 1999. Achieving incremental semantic interpretation through contextual representation. Cognition 71(2). 109147.

Shannon, Claude E. \& Warren Weaver. 1949. The Mathematical Theory of Communication. University of Illinois Press.

Sonnenschein, Susan \& Grover J. Whitehurs. 1984. Developing referential communication-skills the interaction of role-switching and difference rule training. Journal of Experimental Child Psychology 38(2). 191-207.

Sperber, Dan \& Dierdre Wilson. 1986 [1995]. Relevance: Communication and Cognition. Oxford: Blackwell.

Strawson, Peter F. 1950. On Referring. Mind 59. 320-344.

Tanenhaus, Michael K. \& Sarah Brown-Schmidt. 2008. Language Processing in the natural world. Philosophical Transactions of the Royal Society 363. 1105-1122.

Van Deemter, K. 2002. Generating referring expressions: Boolean extensions of the Incremental Algorithm. Computational Linguistics 28(1). 37-52.

Van Deemter, Kees, Albert Gatt, Roger PG van Gompel \& Emiel Krahmer. 2009. Production of Referring Expressions (PRE-CogSci): Bridging the gap between computational and empirical approaches to reference. Proceedings of the $31^{\text {st }}$ Annual Meeting of the Cognitive Science Society (CogSci 2009), Amsterdam, 29 July-1 August 2009. 
Van der Sluis, lelka \& Emiel Krahmer. 2007. Generating multimodal references. Discourse Processes 44. 145-174.

Viethen, Jette \& Robert Dale. 2006. Algorithms for generating referring expressions: Do they do what people do?. Proceedings of the Fourth International Natural Language Generation Conference, Sydney, July 2006. 63-70.

Viethen, Jette \& Robert Dale. 2008. Generating Relational References: What Makes a Difference?. Proceedings of the 2008 Australasian Language Technology Workshop, CSIRO Tasmania ICT Centre, Hobart, December 2008.

Viethen, Jette, Robert Dale, E. J. Krahmer, Mariët Theune \& Pascal Touset. 2008. Controlling redundancy in referring expressions. Proceedings of the $6^{\text {th }}$ Language Resources and Evaluation Conference, Marrakech, May 2008. 